\title{
Effects of LCZ696 (Sacubitril/Valsartan) on Blood Pressure in Patients with Hypertension: A Meta-Analysis of Randomized Controlled Trials
}

\author{
Qiang Geng Rongqiang Yan Zhengzhong Wang Fangjie Hou
}

Department of Cardiology, Qingdao Municipal Hospital, Qingdao, China

\section{Keywords}

LCZ696 · RAS inhibition · Blood pressure · Hypertension ·

Meta-analysis

\begin{abstract}
Background: LCZ696 (sacubitril/valsartan), a first-in-class angiotensin receptor-neprilysin inhibitor, can significantly reduce blood pressure in patients with heart failure. We performed this meta-analysis to determine the antihypertensive effect of LCZ696 in patients with hypertension. Methods: Randomized controlled trials were searched in MEDLINE, the Cochrane Library, and Clinicaltrials.gov. Twelve studies with a total of 6,064 participants were included. $\boldsymbol{R} \boldsymbol{e}$ sults: Compared with angiotensin receptor blockers (ARBs), LCZ696 100 mg caused a significant reduction in systolic blood pressure (SBP) (mean difference [MD] $-1.58 \mathrm{~mm} \mathrm{Hg}$, $95 \%$ confidence interval $[\mathrm{Cl}]-2.09$ to $-1.07, p<0.05)$ and diastolic blood pressure (DBP) (MD $-0.66 \mathrm{~mm} \mathrm{Hg}, 95 \% \mathrm{Cl}$ -0.98 to $-0.33, p<0.05)$. LCZ696 $200 \mathrm{mg}$ caused a significant reduction in SBP (MD $-4.94 \mathrm{~mm} \mathrm{Hg}, 95 \% \mathrm{Cl}-6.54$ to -3.35 , $p<0.05$ ), DBP (MD $-2.24 \mathrm{~mm} \mathrm{Hg}, 95 \% \mathrm{Cl}-2.74$ to -1.75 , $p<0.05)$, 24-h ambulatory SBP (24h ASBP; MD -3.69 mm Hg, $95 \% \mathrm{Cl}-4.80$ to $-2.58, p<0.05)$, and 24-h ADBP (MD -1.71 $\mathrm{mm} \mathrm{Hg}, 95 \% \mathrm{Cl}-2.13$ to $-1.28, p<0.05)$. LCZ696 $400 \mathrm{mg}$
\end{abstract}

caused a significant reduction in SBP (MD $-6.25 \mathrm{~mm} \mathrm{Hg}, 95 \%$ $\mathrm{Cl}-7.90$ to $-4.61, p<0.05$ ), DBP (MD $-2.30 \mathrm{~mm} \mathrm{Hg}, 95 \% \mathrm{Cl}$ -2.80 to $-1.80, p<0.05$ ), 24-h ASBP (MD $-4.31 \mathrm{~mm} \mathrm{Hg}, 95 \%$ $\mathrm{Cl}-6.56$ to $-2.07, p<0.05)$, and $24 \mathrm{~h}$ ADBP (MD $-1.69 \mathrm{~mm} \mathrm{Hg}$, $95 \% \mathrm{Cl}-2.59$ to $-0.79, p<0.05)$. Compared with LCZ696 200 $\mathrm{mg}, \mathrm{LCZ696} 400 \mathrm{mg}$ caused a significant reduction in SBP (MD $1.71 \mathrm{~mm} \mathrm{Hg}, 95 \% \mathrm{Cl} 1.15$ to 2.27, $p<0.05$ ), DBP (MD 0.90 $\mathrm{mm} \mathrm{Hg}, 95 \% \mathrm{Cl} 0.65$ to $1.16, p<0.05)$, 24-h ASBP (MD 1.50 $\mathrm{mm} \mathrm{Hg}, 95 \% \mathrm{Cl} 0.84$ to $2.17, p<0.05$ ), and 24-h ADBP (MD $0.76 \mathrm{~mm} \mathrm{Hg}, 95 \% \mathrm{Cl} 0.47$ to $1.06, p<0.05)$. Conclusions: The blood pressure-lowering effect of LCZ696 is dose-related. This meta-analysis confirms the antihypertensive effects of LCZ696.

(c) 2020 S. Karger AG, Basel

\section{Introduction}

Neprilysin degrades vasoactive peptides, including natriuretic peptides and bradykinin [1]. Neprilysin inhibitors are expected to increase the levels of these substances and reduce blood pressure. Combined inhibitors of the renin-angiotensin system (RAS) and neprilysin are superior to those of either approach alone in reducing blood pressure [2]. Sacubitril/valsartan (LCZ696), a first-in-

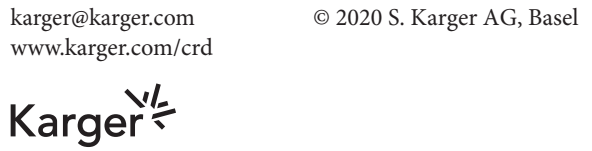


Fig. 1. Flow diagram of the study selection. In total, 1,886 studies were identified. Finally, 12 studies were selected.

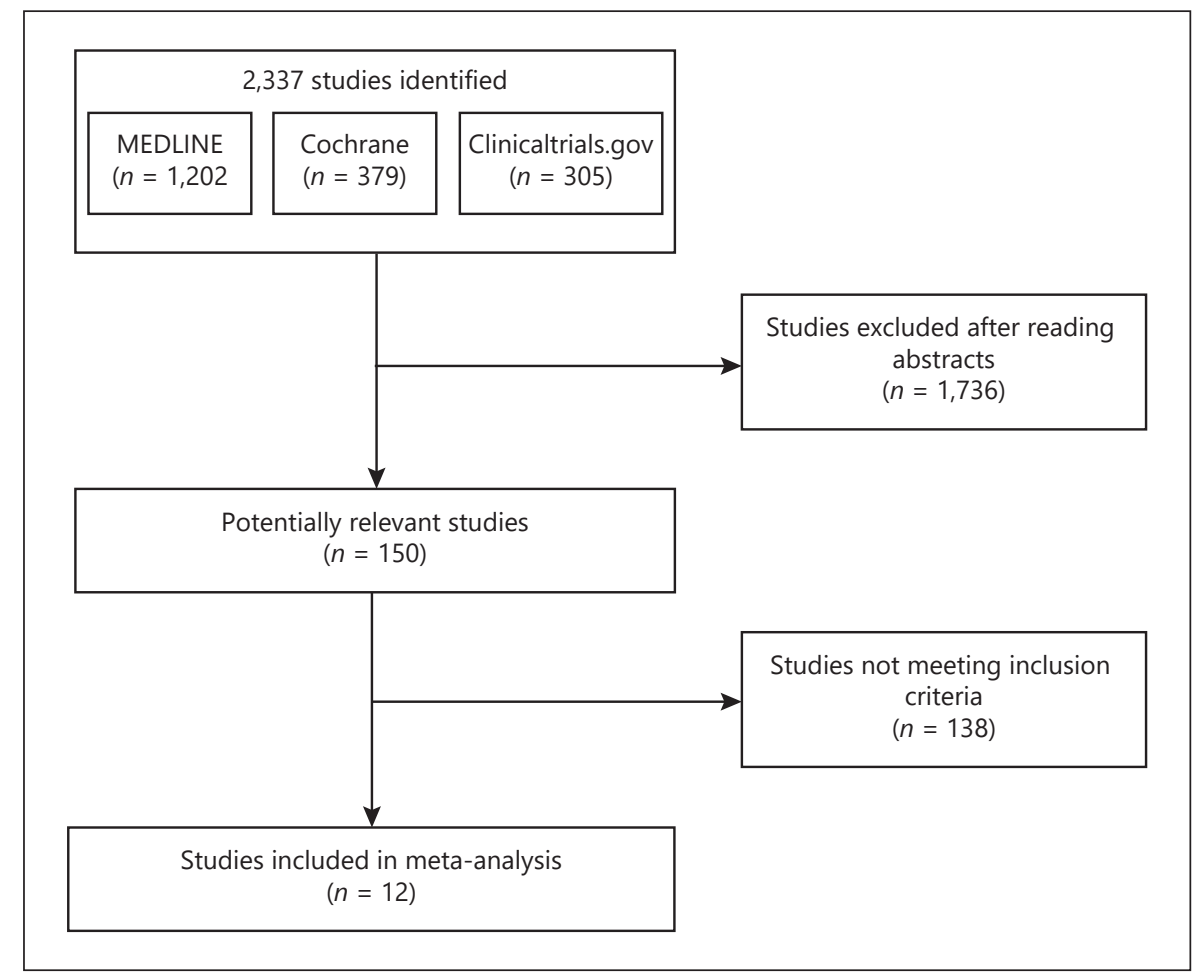

class angiotensin receptor-neprilysin inhibitor, can simultaneously block RAS activation and natriuretic peptide degradation, resulting in greater antihypertensive efficacy [2]. LCZ696 has been approved for the treatment of patients with heart failure and was granted a Class IB recommendation in the $\mathrm{ACC} / \mathrm{AHA}$ and $\mathrm{ESC}$ guidelines $[3,4]$. Can LCZ696 be used as an antihypertensive agent because of its significant antihypertensive effect? This meta-analysis was performed to evaluate the antihypertensive effect of LCZ696 in patients with hypertension.

\section{Methods}

\section{Search Strategy}

We followed the Preferred Reporting Items for Systematic Reviews and Meta-Analyses (PRISMA) guidelines to report this meta-analysis [5]. MEDLINE, the Cochrane Library of Trials, and Clinicaltrials.gov were searched to identify published or unpublished randomized controlled trials (RCTs) from September 1999 to July 2019. The following terms were included: neprilysin inhibitor, LCZ696, sacubitril-valsartan, Entresto.

\section{Inclusion and Exclusion Criteria}

All included studies were RCTs of LCZ696 versus angiotensin receptor blockers (ARBs) in patients with hypertension. The studies reported systolic blood pressure (SBP), diastolic blood pressure
(DBP), 24-h ambulatory systolic blood pressure (24 h ASBP), 24-h ambulatory diastolic blood pressure (24 h ADBP), or a change from baseline in blood pressure. Reviews, case reports, and abstracts were excluded.

\section{Quality Assessment and Data Extraction}

According to the Cochrane Reviewer's Handbook, 2 reviewers (Q.G. and F.H.) independently extracted data from eligible studies. Discrepancies were resolved by discussion. The risk of bias was estimated by using the parameters: sequence generation, concealment of group allocation, blinding during outcome assessment, selective reporting, and intention-to-treat analysis. A flow diagram is shown in Figure 1.

The following terms were extracted: publication year, study design, blinding method, age, total number, duration of therapy, change from the baseline in SBP and DBP, change from the baseline in 24-h ASBP and 24-h ADBP.

Twelve studies reported SBP and DBP [6-17]. Eight studies reported $24 \mathrm{~h} \mathrm{ASBP}$ and $24 \mathrm{~h}$ ADBP [6-13].

\section{Statistical Analysis}

All meta-analyses were performed in Review Manager software (v5.3; Cochrane Collaboration, Oxford, UK). A two-tailed $p$ value $<0.05$ was considered statistically significant. The heterogeneity of studies was assessed with the $I^{2}$ statistic. $I^{2}>50 \%$ and $p<0.10$ indicated statistically significant heterogeneity between studies. A funnel plot was used to assess publication bias. The change from baseline in blood pressure was presented as the mean difference (MD) with a $95 \%$ confidence interval (CI). 
Table 1. Characteristics of the included trials

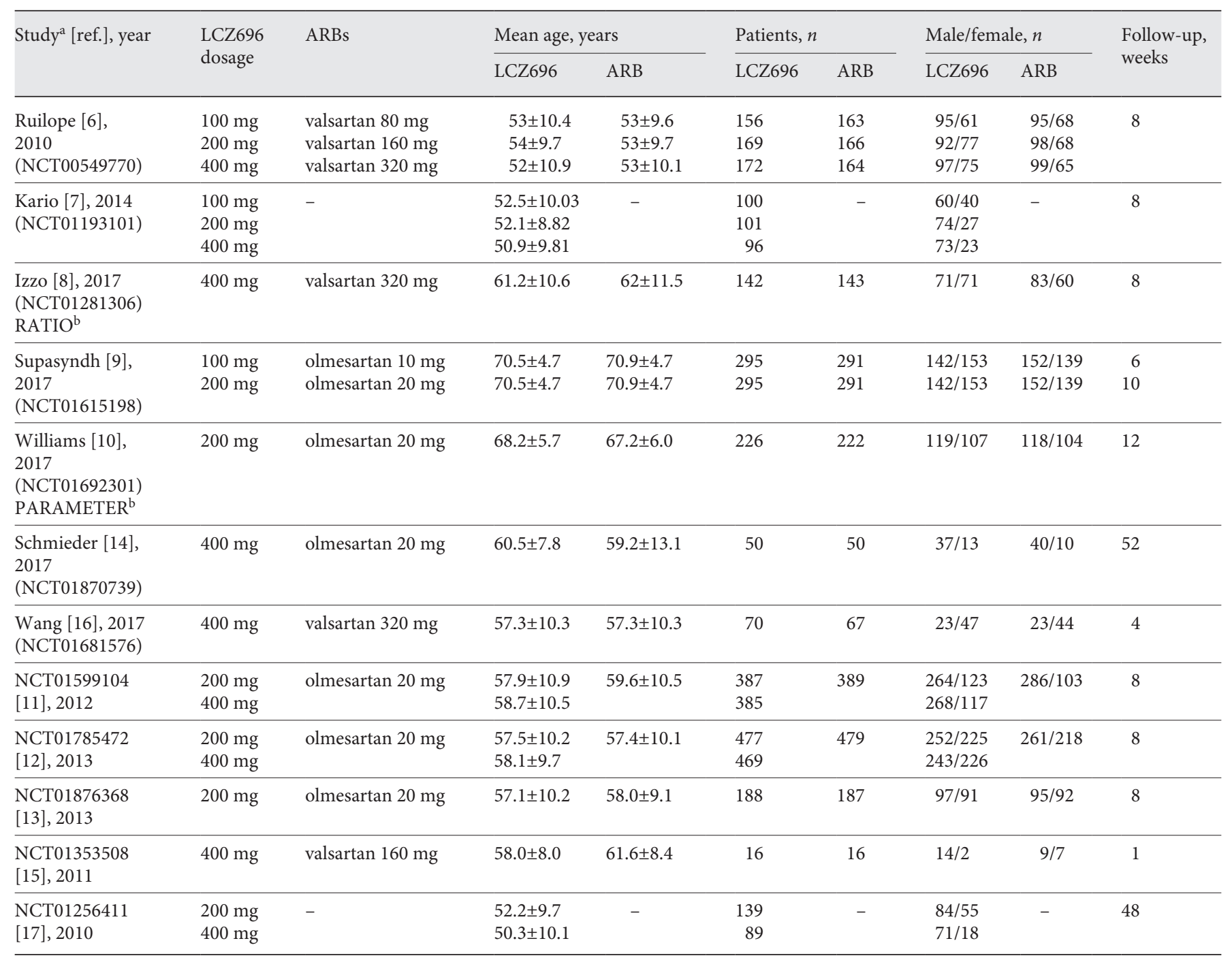

-, No report; ARB, angiotensin receptor blockers. ${ }^{a}$ The ClinicalTrials.gov Identifier is supplied, and, if available, the first author. ${ }^{\mathrm{b}}$ Name of the trial.

\section{Results}

In total, 2,337 studies were identified in MEDLINE, the Cochrane Library of Trials, and Clinicaltrials.gov. Finally, 12 studies with a total of 6,064 participants were selected. Many studies (e.g., PARADIGM-HF, PARAMOUNT, and PIONEER) did not report blood pressure values and were thus not included in our analysis. The baseline characteristics of the included studies appear in Table 1.

Baseline and final SBP levels of LCZ696 and ARBs appear in online supplemental Table 2 (for all online suppl. material, see www.karger.com/doi/10.1159/000507327), and baseline and final DBP levels of LCZ696 and ARBs in online supplemental Table 3. Risk of bias is summarized in online supplemental Table 1. All studies used a parallel-group design. All studies in our analysis are RCTs.

Two studies [6, 9] reported SBP and DBP in LCZ696 $100 \mathrm{mg}$ and ARBs. Compared with ARBs, LCZ696 100 $\mathrm{mg}$ caused a significant reduction in SBP (MD $-1.58 \mathrm{~mm}$ $\mathrm{Hg}, 95 \% \mathrm{CI}-2.09$ to $-1.07, p<0.05)$ and DBP (MD -0.66 $\mathrm{mm} \mathrm{Hg}, 95 \% \mathrm{CI}-0.98$ to $-0.33, p<0.05$ ) (Fig. 2).

Six studies [6, 9-13] reported SBP, DBP, 24 h ASBP, and $24 \mathrm{~h} \mathrm{ADBP}$ in LCZ696 $200 \mathrm{mg}$ and ARBs. Compared with ARBs, LCZ696 $200 \mathrm{mg}$ caused a significant reduction in SBP (MD $-4.94 \mathrm{~mm} \mathrm{Hg}, 95 \% \mathrm{CI}-6.54$ to -3.35 , $p<0.05$ ), DBP (MD $-2.24 \mathrm{~mm} \mathrm{Hg}, 95 \% \mathrm{CI}-2.74$ to -1.75 , 
$p<0.05$ ), $24 \mathrm{~h}$ ASBP (MD $-3.69 \mathrm{~mm} \mathrm{Hg}, 95 \% \mathrm{CI}-4.80$ to $-2.58, p<0.05$ ), and $24 \mathrm{~h} \mathrm{ADBP}(\mathrm{MD}-1.71 \mathrm{~mm} \mathrm{Hg}, 95 \%$ CI -2.13 to $-1.28, p<0.05$ ) (Fig. 3 ).

Seven studies $[6,8,11,12,14-16]$ reported SBP and DBP in LCZ696 $400 \mathrm{mg}$ and ARBs. Four studies $[6,8,11$, 12] reported $24 \mathrm{~h}$ ASBP and $24 \mathrm{~h}$ ADBP in LCZ696 $400 \mathrm{mg}$ and ARBs. Compared with ARBs, LCZ696 $400 \mathrm{mg}$ caused a significant reduction in SBP (MD $-6.25 \mathrm{~mm} \mathrm{Hg}, 95 \% \mathrm{CI}$ -7.90 to $-4.61, p<0.05$ ), DBP (MD $-2.30 \mathrm{~mm} \mathrm{Hg}$, 95\% CI -2.80 to $-1.80, p<0.05), 24 \mathrm{~h}$ ASBP (MD $-4.31 \mathrm{~mm} \mathrm{Hg}$, $95 \% \mathrm{CI}-6.56$ to $-2.07, p<0.05)$, and $24 \mathrm{~h} \mathrm{ADBP}(\mathrm{MD}-1.69$ $\mathrm{mm} \mathrm{Hg}, 95 \% \mathrm{CI}-2.59$ to $-0.79, p<0.05$ ) (Fig. 4 ).

Two studies [6,7] reported SBP, DBP, $24 \mathrm{~h}$ ASBP, and $24 \mathrm{~h}$ ADBP in LCZ696 $100 \mathrm{mg}$ and $200 \mathrm{mg}$. There were no significant differences in SBP (MD $2.83 \mathrm{~mm} \mathrm{Hg}, 95 \%$ CI -1.32 to $6.99, p<0.05$ ) and DBP (MD $1.20 \mathrm{~mm} \mathrm{Hg}$, $95 \%$ CI -2.23 to $4.63, p<0.05)$. Compared with LCZ696 $100 \mathrm{mg}$, LCZ696 $200 \mathrm{mg}$ caused a significant reduction in $24 \mathrm{~h}$ ASBP (MD $2.9 \mathrm{~mm} \mathrm{Hg}$, 95\% CI 1.34 to $4.45, p<0.05$ ), but the MD of $24 \mathrm{~h} \mathrm{ADBP}$ was not statistically significant between LCZ696 $100 \mathrm{mg}$ and $200 \mathrm{mg}$ (MD $1.98 \mathrm{~mm} \mathrm{Hg}$, $95 \%$ CI 0.03 to $3.93, p=0.05$ ) (Fig. 5).

Five studies $[6,7,11,12,17]$ reported SBP and DBP in LCZ696 $200 \mathrm{mg}$ and $400 \mathrm{mg}$. Four studies $[6,7,11,12]$ reported $24 \mathrm{~h}$ ASBP and $24 \mathrm{~h}$ ADBP in LCZ696 $200 \mathrm{mg}$ and $400 \mathrm{mg}$. Compared with LCZ696200 mg, LCZ696 400 mg caused a significant reduction in SBP (MD $1.71 \mathrm{~mm}$ $\mathrm{Hg}$, 95\% CI 1.15 to 2.27, $p<0.05$ ), DBP (MD $0.90 \mathrm{~mm} \mathrm{Hg}$, $95 \%$ CI 0.65 to $1.16, p<0.05$ ), $24 \mathrm{~h}$ ASBP (MD $1.50 \mathrm{~mm}$ $\mathrm{Hg}$, 95\% CI 0.84 to $2.17, p<0.05$ ), and $24 \mathrm{~h} \mathrm{ADBP}$ (MD $0.76 \mathrm{~mm} \mathrm{Hg}$, 95\% CI 0.47 to $1.06, p<0.05$ ) (Fig. 6).

\section{Discussion}

Currently, the available evidence shows that LCZ696 can provide greater benefits for heart failure patients [1820]. There has been little research into LCZ696 as an an-

SBP

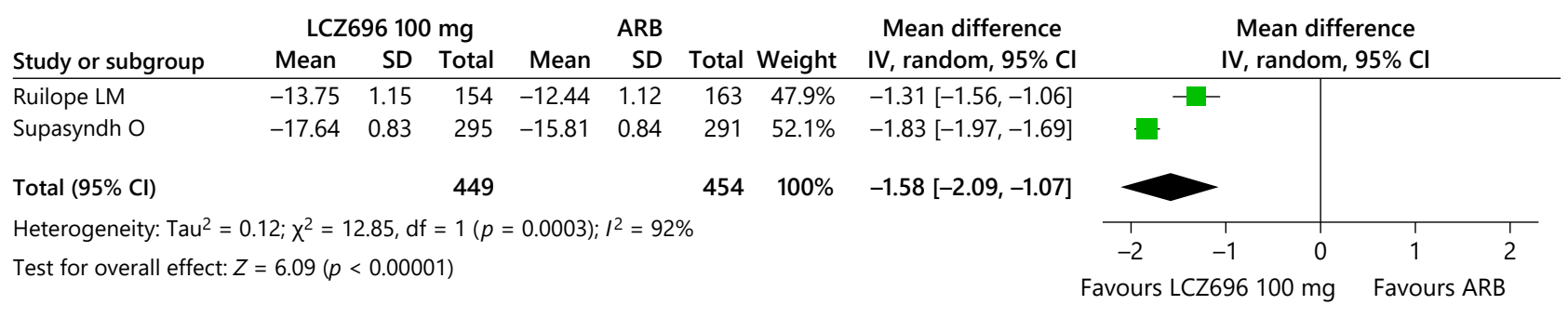

DBP

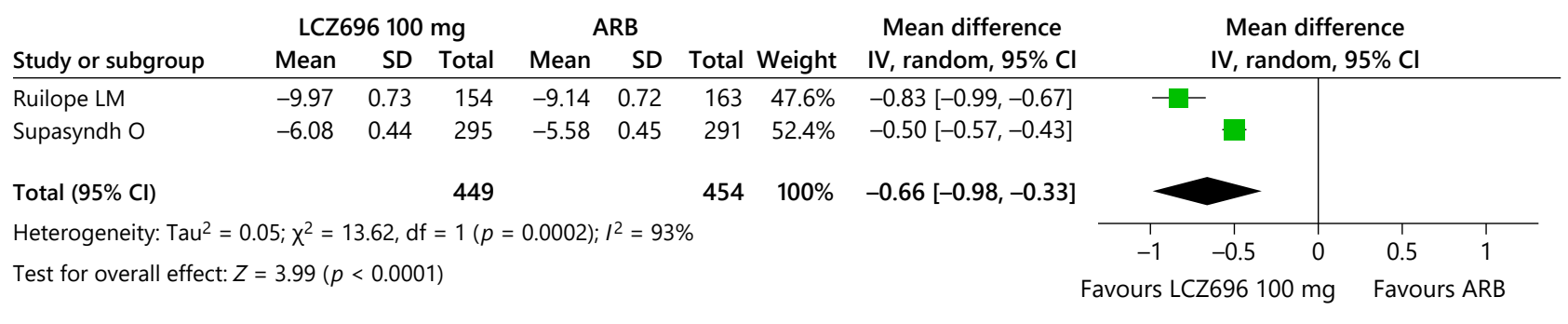

Fig. 2. Forest plots of SBP and DBP in LCZ696 $100 \mathrm{mg}$ versus ARBs. LCZ696 $100 \mathrm{mg}$ showed a significant reduction in SBP (MD $-1.58 \mathrm{~mm} \mathrm{Hg}, 95 \% \mathrm{CI}-2.09$ to $-1.07, p<0.05)$ and DBP (MD $-0.66 \mathrm{~mm} \mathrm{Hg}, 95 \% \mathrm{CI}-0.98$ to $-0.33, p<0.05)$ when compared with ARBs.

Fig. 3. Forest plots of SBP, DBP, $24 \mathrm{~h} \mathrm{ASBP}$, and $24 \mathrm{~h}$ ADBP in LCZ696 $200 \mathrm{mg}$ versus ARBs. LCZ696 $200 \mathrm{mg}$ showed a significant reduction in SBP (MD $-4.94 \mathrm{~mm} \mathrm{Hg}, 95 \% \mathrm{CI}-6.54$ to $-3.35, p<0.05)$, DBP (MD $-2.24 \mathrm{~mm}$ $\mathrm{Hg}, 95 \% \mathrm{CI}-2.74$ to $-1.75, p<0.05$ ), $24 \mathrm{~h} \mathrm{ASBP}(\mathrm{MD}-3.69 \mathrm{~mm} \mathrm{Hg}, 95 \% \mathrm{CI}-4.80$ to $-2.58, p<0.05)$, and $24 \mathrm{~h}$ ADBP (MD $-1.71 \mathrm{~mm} \mathrm{Hg}, 95 \% \mathrm{CI}-2.13$ to $-1.28, p<0.05)$ when compared with ARBs.

(For figure see next page.) 
SBP

LCZ696 $200 \mathrm{mg} \quad$ ARB

Study or subgroup Mean SD Total Mean SD Total Weight

Mean difference

Mean difference

$\begin{array}{llllllll}\text { Supasyndh O } & -22.71 & 0.91 & 295 & -16.11 & 0.92 & 291 & 16.7 \%\end{array}$

Ruliope LM

$\begin{array}{lllllll}-18.7 & 1.1 & 168 & -13.42 & 1.12 & 163 & 16.6 \%\end{array}$

PARAMETER

$-20.84 \quad 1.06$

$\begin{array}{lll}226 & -14.57 & 1.07\end{array}$

$222 \quad 16.7 \%$

NCT01876368

$\begin{array}{ll}-14.21 & 1.28\end{array}$

$\begin{array}{lllll}188 & -10.03 & 1.29 & 187 & 16.6 \%\end{array}$

NCT01785472

$\begin{array}{lllllll}-20.48 & 0.61 & 477 & -18.15 & 0.61 & 479 & 16.7 \%\end{array}$

NCT01599104

$\begin{array}{lllllll}-18.21 & 0.7 & 387 & -13.2 & 0.7 & 389 & 16.7 \%\end{array}$

Total $(95 \% \mathrm{Cl})$

1,741

$1,731 \quad 100 \%$

Heterogeneity: $\mathrm{Tau}^{2}=3.96 ; \chi^{2}=3,991.13, \mathrm{df}=5(p<0.00001) ; l^{2}=100 \%$

Test for overall effect: $Z=6.08(p<0.00001)$

IV, random, $95 \% \mathrm{Cl}$

IV, random, $95 \% \mathrm{Cl}$

$-6.60[-6.75,-6.45]$

$-5.28[-5.52,-5.04]$

$-6.27[-6.47,-6.07]$

$-4.18[-4.44,-3.92]$

$-2.33[-2.41,-2.25]$

$-5.01[-5.11,-4.91]$

$-4.94[-6.54,-3.35]$

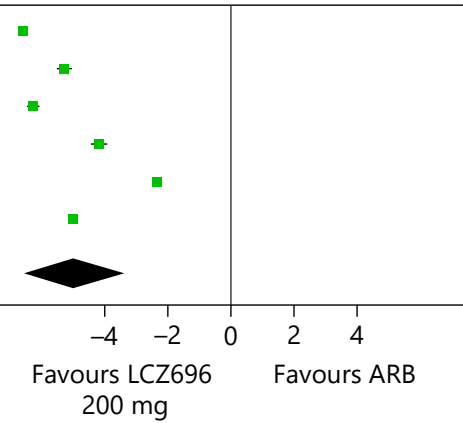

DBP

Study or subgroup

LCZ696 $200 \mathrm{mg} \quad$ ARB

Mean difference

Supasyndh O

Mean SD Total Mean SD Total Weight

IV, random, $95 \% \mathrm{Cl}$

Mean difference

Ruliope LM

$\begin{array}{lllllll}-8.58 & 0.47 & 295 & -6.49 & 0.48 & 291 & 16.7 \%\end{array}$

$-2.09[-2.17,-2.01]$

$-2.97[-3.12,-2.82]$

$-2.28[-2.39,-2.17]$

PARAMETER

$\begin{array}{lllllll}-12.92 & 0.7 & 168 & -9.95 & 0.71 & 163 & 16.5 \% \\ -7.86 & 0.58 & 226 & -5.58 & 0.59 & 222 & 16.7 \%\end{array}$

NCT01876368

$\begin{array}{llllllll}-7.52 & 0.7 & 188 & -4.47 & 0.71 & 187 & 16.6 \%\end{array}$

NCT01785472

$\begin{array}{lllllll}-8.1 & 0.37 & 477 & -6.86 & 0.37 & 479 & 16.8 \%\end{array}$

NCT01599104

$\begin{array}{lllllll}-7.76 & 0.4 & 387 & -5.91 & 0.4 & 389 & 16.8 \%\end{array}$

Total $(95 \% \mathrm{Cl})$

1,741

$1,731 \quad 100 \%$

$-3.05[-3.19,-2.91]$

$-1.24[-1.29,-1.19]$

$-1.85[-1.91,-1.79]$

$-2.24[-2.74,-1.75]$

Heterogeneity: $\mathrm{Tau}^{2}=0.39 ; \chi^{2}=1,198.32, \mathrm{df}=5(p<0.00001) ; R^{2}=100 \%$

Test for overall effect: $Z=8.82(p<0.00001)$

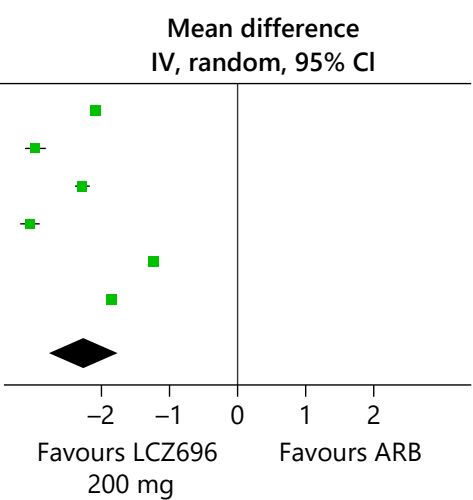

\section{$24 \mathrm{~h}$ ASBP}

\begin{tabular}{lccrrrrrr} 
& \multicolumn{2}{c}{ LCZ696 200} & $\mathbf{m g}$ & \multicolumn{2}{c}{ ARB } \\
Study or subgroup & Mean & SD & Total & Mean & SD & Total & Weight \\
\hline Supasyndh O & -14.23 & 0.56 & 154 & -9.14 & 0.56 & 157 & $16.7 \%$ \\
Ruliope LM & -11.5 & 0.93 & 61 & -8.27 & 1 & 49 & $16.4 \%$ \\
PARAMETER & -13.25 & 0.62 & 164 & -9.14 & 0.62 & 162 & $16.7 \%$ \\
NCT01876368 & -4.26 & 0.6 & 167 & -1.04 & 0.61 & 164 & $16.7 \%$ \\
NCT01785472 & -12.07 & 0.49 & 188 & -10.26 & 0.49 & 182 & $16.7 \%$ \\
NCT01599104 & -13.44 & 0.45 & 216 & -8.78 & 0.46 & 200 & $16.7 \%$ \\
Total $(95 \% \mathrm{Cl})$ & & & 950 & & & 914 & $100 \%$
\end{tabular}

Heterogeneity: $\mathrm{Tau}^{2}=1.92 ; \chi^{2}=2,408.28, \mathrm{df}=5(p<0.00001) ; R^{2}=100 \%$

Test for overall effect: $Z=6.51(p<0.00001)$

IV, random, $95 \% \mathrm{Cl}$

$-5.09[-5.21,-4.97]$

$-3.23[-3.59,-2.87]$

$-4.11[-4.24,-3.98]$

$-3.22[-3.35,-3.09]$

$-1.81[-1.91,-1.71]$

$-4.66[-4.75,-4.57]$

$-3.69[-4.80,-2.58]$ $200 \mathrm{mg}$

\section{$24 \mathrm{~h}$ ADBP}

Study or subgroup Mean SD Total Mean SD Total Weight

Mean difference

IV, random, $95 \% \mathrm{Cl}$

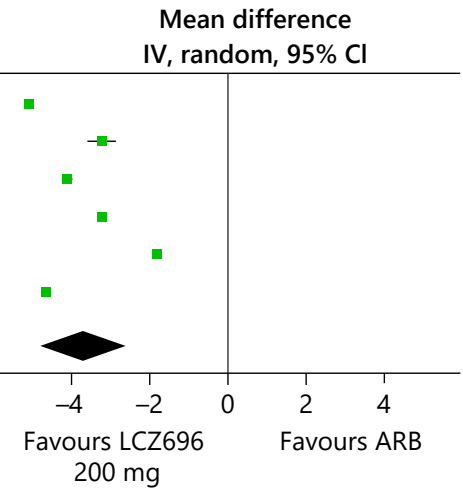

\begin{tabular}{lrlrllll}
\hline Supasyndh O & -6.95 & 0.31 & 154 & -4.47 & 0.31 & 157 & $16.8 \%$ \\
Ruliope LM & -6.78 & 0.64 & 61 & -6.25 & 0.7 & 155 & $16.0 \%$ \\
PARAMETER & -7.44 & 0.37 & 164 & -5.48 & 0.36 & 162 & $16.8 \%$ \\
NCT01876368 & -2.27 & 0.39 & 167 & -0.35 & 0.39 & 164 & $16.8 \%$ \\
NCT01785472 & -6.36 & 0.31 & 188 & -5.16 & 0.31 & 182 & $16.8 \%$ \\
NCT01599104 & -7.65 & 0.3 & 216 & -5.56 & 0.31 & 200 & $16.8 \%$ \\
Total $(95 \% \mathrm{Cl})$ & & & 950 & & & 920 & $100 \%$
\end{tabular}

Heterogeneity: $\mathrm{Tau}^{2}=0.28 ; \chi^{2}=906.19, \mathrm{df}=5(p<0.00001) ; l^{2}=99 \%$

Test for overall effect: $Z=7.89(p<0.00001)$

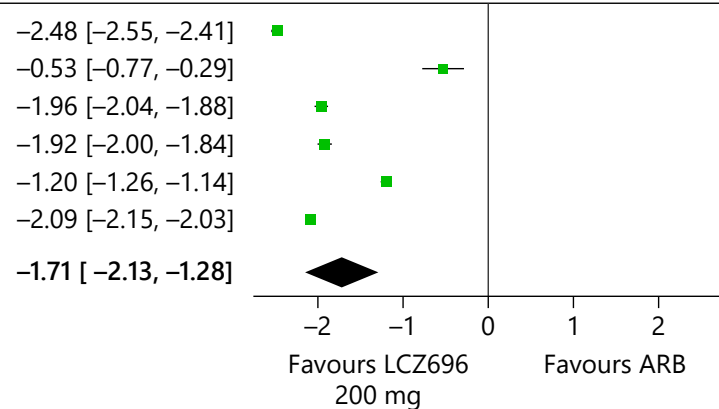




\section{SBP}

LCZ696 $400 \mathrm{mg}$

Study or subgroup Mean SD Total

$A R B$

Mean SD Total Weight

Wang TD

$\begin{array}{lllllll}-13.19 & 0.97 & 70 & -5.78 & 2.46 & 67 & 15.0 \%\end{array}$

Schmieder RE

$\begin{array}{lllllll}-16.7 & 1.5 & 50 & -13.6 & 1.5 & 50 & 15.0 \%\end{array}$

Ruilope LM

RATIO

NCT01785472

$-20.17 \quad 1.1$

$\begin{array}{lll}170 & -14.16 & 1.12\end{array}$

$163 \quad 15.3 \%$

$\begin{array}{llllllll}-21.8 & 1.22 & 142 & -16.1 & 1.22 & 143 & 15.2 \%\end{array}$

$\begin{array}{lllllll}-21.67 & 0.62 & 469 & -18.15 & 0.61 & 479 & 15.3 \%\end{array}$

NCT01599104

NCT01353508

$\begin{array}{llllllll}-20.18 & 0.7 & 385 & -13.2 & 0.7 & 389 & 15.3 \%\end{array}$

$\begin{array}{lllllll}-15.8 & 6.2 & 16 & -1.3 & 3.92 & 16 & 8.9 \%\end{array}$

Total $(95 \% \mathrm{Cl})$

1,302

$1,307 \quad 100 \%$

Heterogeneity: Tau $^{2}=4.61 ; \chi^{2}=3,134.12, \mathrm{df}=6(p<0.00001) ; R^{2}=100 \%$

Test for overall effect: $Z=7.44(p<0.00001)$

Mean difference Mean difference

IV, random, $95 \% \mathrm{Cl}$

IV, random, $95 \% \mathrm{Cl}$

$-7.41[-8.04,-6.78]$

$-3.10[-3.69,-2.51]$

$-6.01[-6.25,-5.77]$

$-5.70[-5.98,-5.42]$

$-3.52[-3.60,-3.44]$

$-6.98[-7.08,-6.88]$

$-14.50[-18.09,-10.91]$

$-6.25[-7.90,-4.61]$

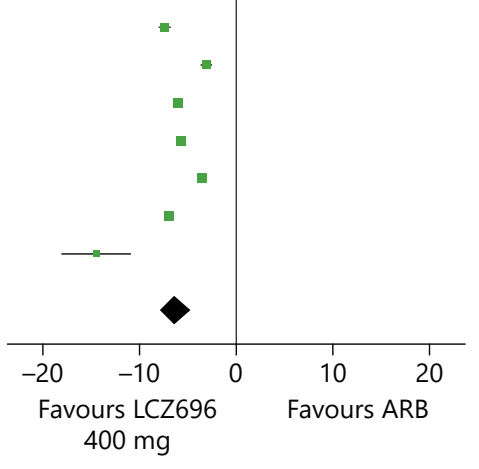

DBP
LCZ696 400 mg Mean SD Total

Study or subgroup

Wang TD

Schmieder RE

Ruilope LM

RATIO

NCT01785472

NCT01599104

NCT01353508

Total $(95 \% \mathrm{Cl})$

Test for overall effect: $Z=8.95(p<0.00001)$
Heterogeneity: $\operatorname{Tau}^{2}=0.42 ; \chi^{2}=805.62, \mathrm{df}=$

$\begin{array}{llllllll}-6.19 & 1.11 & 70 & -4.19 & 0.02 & 67 & 15.2 \%\end{array}$

$\begin{array}{lllllll}-10.3 & 1.06 & 50 & -10.4 & 1.06 & 50 & 14.3 \%\end{array}$

$\begin{array}{lllllll}-13.63 & 0.7 & 170 & -10.93 & 0.71 & 163 & 15.6 \%\end{array}$

$\begin{array}{llllllll}-9.6 & 0.75 & 142 & -7.3 & 0.76 & 143 & 15.5 \%\end{array}$

$\begin{array}{lllllll}-8.8 & 0.38 & 469 & -6.86 & 0.37 & 479 & 15.8 \%\end{array}$

$\begin{array}{llllllll}-8.79 & 0.41 & 385 & -5.91 & 0.4 & 389 & 15.8 \%\end{array}$

$\begin{array}{lllllll}-6.9 & 2.21 & 16 & -0.8 & 1.48 & 16 & 7.7 \%\end{array}$

$1,302 \quad 1,307 \quad 100 \%$

1,307
$R=99 \%$

.

\section{$24 \mathrm{~h}$ ASBP}

Study or subgroup

Ruilope LM

RATIO

NCT01785472

NCT01599104

Total $(95 \% \mathrm{Cl})$

LCZ696 $400 \mathrm{mg}$

$\begin{array}{rrr}\text { Mean } & \text { SD } & \text { Total } \\ -14.56 & 1.03 & 53\end{array}$

$\begin{array}{lll}-13 & 0.71\end{array}$

$\begin{array}{ll}-12.76 & 0.49\end{array}$

$-14.99 \quad 0.45$

Heterogeneity: Tau $^{2}=5.23 ; \chi^{2}=3,110.25, \mathrm{df}=3(p<0.00001) ; R^{2}=100 \%$

Test for overall effect: $Z=3.77(p=0.0002)$

\begin{tabular}{lrrr}
\multicolumn{2}{c}{ ARB } & & \\
Mean & SD & Total & Weight \\
-9.42 & 0.97 & 54 & $24.9 \%$ \\
-9.6 & 0.71 & 93 & $25.0 \%$ \\
-10.26 & 0.49 & 182 & $25.1 \%$ \\
-8.78 & 0.46 & 200 & $25.1 \%$ \\
& & 529 & $100 \%$
\end{tabular}

$$
\begin{aligned}
& \text { Me } \\
& \text { IV, } \\
& \hline-2 \\
& 0 \\
& -2 \\
& -2 \\
& -1 \\
& -2 \\
& -6 . \\
& -2 \\
&
\end{aligned}
$$

Mean difference Mean difference

IV, random, $95 \% \mathrm{Cl} \quad \mathrm{IV}$, random, $95 \% \mathrm{Cl}$

$-2.00[-2.26,-1.74]$

$0.10[-0.32,0.52]$

$-2.70[-2.85,-2.55]$

$-2.30[-2.48,-2.12]$

$-1.94[-1.99,-1.89]$

$-2.88[-2.94,-2.82]$

$-6.10[-7.40,-4.80]$

$-2.30[-2.80,-1.80]$

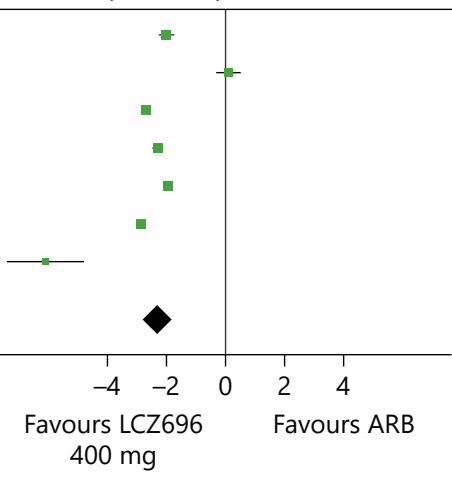

Mean difference Mean difference

IV, random, $95 \% \mathrm{Cl} \quad \mathrm{IV}$, random, $95 \% \mathrm{Cl}$

$-5.14[-5.52,-4.76]$

$-3.40[-3.61,-3.19]$

$-2.50[-2.60,-2.40]$

$-6.21[-6.30,-6.12]$

$-4.31[-6.56,-2.07]$

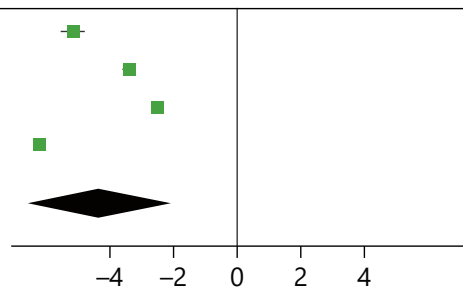

Favours LCZ696 Favours ARB $400 \mathrm{mg}$

$24 \mathrm{~h}$ ADBP

Study or subgroup Mean SD Total Mean SD Total Weight

Mean difference

IV, random, $95 \% \mathrm{Cl}$

Mean difference

$-1.19[-1.45,-0.93]$

$-1.00[-1.13,-0.87]$

RATIO

NCT01785472

$\begin{array}{lllllll}-8.32 & 0.71 & 53 & -7.13 & 0.67 & 54 & 24.7 \% \\ -6.2 & 0.44 & 90 & -5.2 & 0.44 & 93 & 25.1 \%\end{array}$

NCT01599104

$\begin{array}{lllllll}-6.82 & 0.32 & 184 & -5.16 & 0.31 & 182 & 25.1 \%\end{array}$

$\begin{array}{lllllll}-8.44 & 0.3 & 216 & -5.56 & 0.31 & 200 & 25.2 \%\end{array}$

$-1.66[-1.72,-1.60]$

$-2.88[-2.94,-2.82]$

Total $(95 \% \mathrm{Cl})$

543

$529100 \%$

$-1.69[-2.59,-0.79]$

Heterogeneity: Tau $^{2}=0.84 ; \chi^{2}=1,178.94, \mathrm{df}=3(p<0.00001) ; R^{2}=100 \%$

Test for overall effect: $Z=3.67(p=0.0002)$

IV, random, 95\% Cl

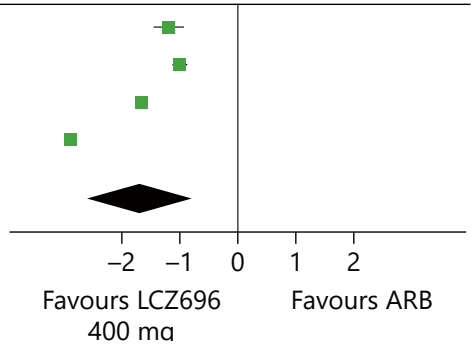


SBP

\begin{tabular}{|c|c|c|c|c|c|c|c|c|}
\hline \multirow{2}{*}{ Study of subgroup } & \multicolumn{3}{|c|}{ LCZ696 $100 \mathrm{mg}$} & \multicolumn{3}{|c|}{ LCZ696 200 mg } & \multirow[b]{2}{*}{ Weight } & \multirow{2}{*}{$\begin{array}{l}\text { Mean difference } \\
\text { IV random, } 95 \% \mathrm{Cl}\end{array}$} \\
\hline & Mean & SD & Total & Mean & SD & Total & & \\
\hline Kario K & -16.83 & 1.25 & 100 & -17.54 & 1.27 & 98 & $50.0 \%$ & $0.71[0.36,1.06]$ \\
\hline Ruilope LM & -13.75 & 1.15 & 154 & -18.7 & 1.1 & 168 & $50.0 \%$ & $4.95[4,70,5.20]$ \\
\hline Total $(95 \% \mathrm{Cl})$ & & & 254 & & & 266 & $100 \%$ & $2.83[-1.32,6.99]$ \\
\hline
\end{tabular}

Heterogeneity: $\mathrm{Tau}^{2}=8.96 ; \chi^{2}=375.53, \mathrm{df}=1(p<0.00001) ; 1^{2}=100 \%$

Test for overall effect: $Z=1.34(p=0.18)$

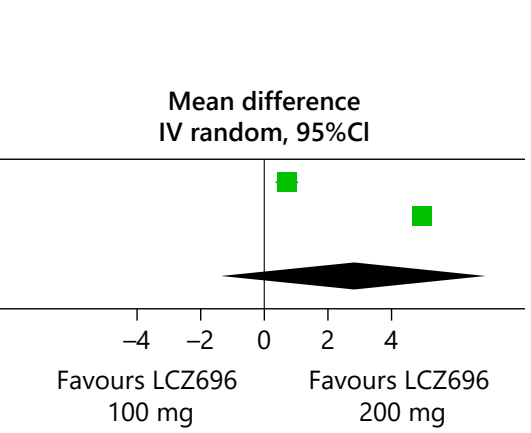

DBP

\begin{tabular}{|c|c|c|c|c|c|c|c|}
\hline \multirow[b]{2}{*}{ Study of subgroup } & \multicolumn{3}{|c|}{ LCZ696 100 mg } & \multicolumn{3}{|c|}{ LCZ696 200 mg } & \multirow[b]{2}{*}{ Weight } \\
\hline & Mean & SD & Total & Mean & SD & Total & \\
\hline Kario K & -11.53 & 0.88 & 100 & -10.98 & 0.89 & 98 & $50.0 \%$ \\
\hline Ruilope LM & -9.97 & 0.73 & 154 & -12.92 & 0.7 & 168 & $50.0 \%$ \\
\hline Total $(95 \% \mathrm{Cl})$ & & & 254 & & & 266 & $100 \%$ \\
\hline
\end{tabular}

Mean difference Mean difference

IV random, $95 \% \mathrm{Cl} \quad$ IV random, $95 \% \mathrm{Cl}$

$-0.55[-0.80,-0.30]$

$2.95[2.79,3.11]$

$1.20[-2.23,4.63]$

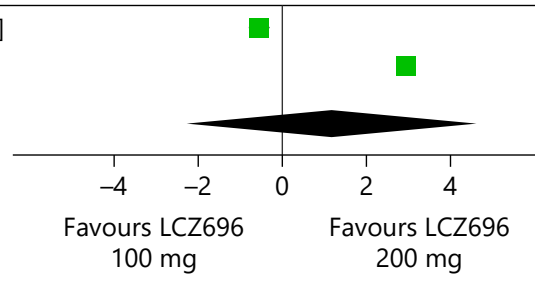

24 h ASBP

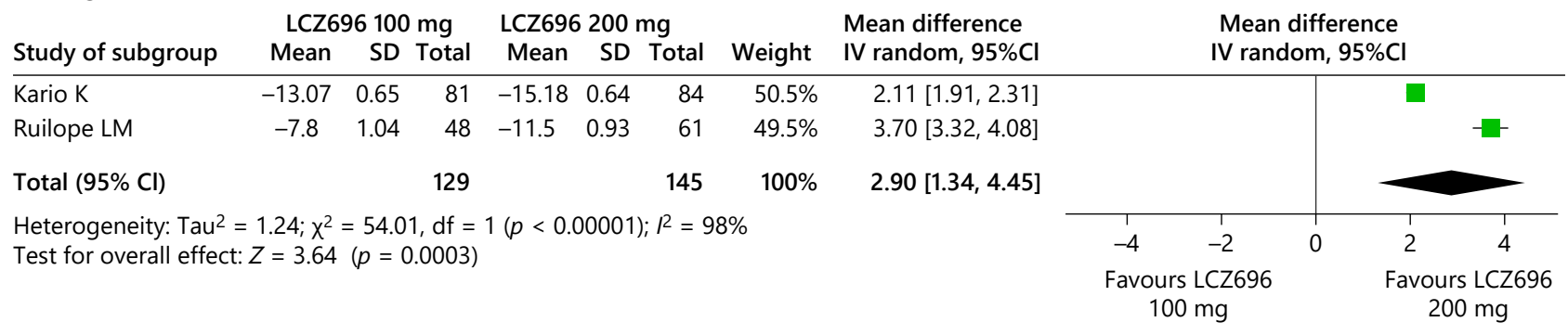

$24 \mathrm{~h}$ ADBP

Study of subgroup

Kario K

LCZ696 $100 \mathrm{mg} \quad$ LCZ696 $200 \mathrm{mg}$

Mean SD Total Mean SD Total Weight

Mean difference

Mean difference

Ruilope LM

$\begin{array}{lllllll}-8.34 & 0.5 & 81 & -9.33 & 0.49 & 84 & 50.1 \%\end{array}$

random, $95 \% \mathrm{Cl}$

IV random, $95 \% \mathrm{Cl}$

Total $(95 \% \mathrm{Cl})$

$\begin{array}{lllllll}-3.8 & 0.72 & 48 & -6.78 & 0.64 & 61 & 49.9 \%\end{array}$

$99[0.84,1.14]$

$2.98[2.72,3.24]$

Heterogeneity: Tau $^{2}=1.97 ; \chi^{2}=168.81, \mathrm{df}=1(p<0.00001) ; R^{2}=99 \%$

Test for overall effect: $Z=1.99(p=0.05)$

$1.98[0.03,3.93]$

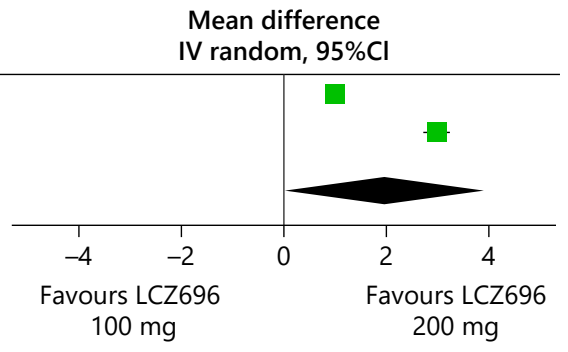

Fig. 5. Forest plots of SBP, DBP, $24 \mathrm{~h} \mathrm{ASBP}$, and 24h ADBP in LCZ696 100 versus $200 \mathrm{mg}$. The MD of SBP (MD $2.83 \mathrm{~mm} \mathrm{Hg}, 95 \% \mathrm{CI}-1.32$ to 6.99, $p<0.05$ ), DBP (MD $1.20 \mathrm{~mm} \mathrm{Hg}, 95 \% \mathrm{CI}-2.23$ to $4.63, p<0.05$ ), and $24 \mathrm{~h}$ ADBP (MD $1.98 \mathrm{~mm} \mathrm{Hg}$, 95\% CI 0.03 to 3.93, $p=0.05$ ) was not statistically significant between 100 and $200 \mathrm{mg}$ LCZ696. LCZ696 $200 \mathrm{mg}$ showed a significant reduction in $24 \mathrm{~h}$ ASBP (MD $2.9 \mathrm{~mm} \mathrm{Hg}$, 95\% CI 1.34 to 4.45, $p<0.05)$.

Fig. 4. Forest plots of SBP, DBP, $24 \mathrm{~h} \mathrm{ASBP}$, and $24 \mathrm{~h}$ ADBP in LCZ696 $400 \mathrm{mg}$ versus ARBs. LCZ696 $400 \mathrm{mg}$ showed a significant reduction in SBP (MD $-6.25 \mathrm{~mm} \mathrm{Hg}, 95 \% \mathrm{CI}-7.90$ to $-4.61, p<0.05)$, DBP (MD $-2.30 \mathrm{~mm}$ $\mathrm{Hg}, 95 \% \mathrm{CI}-2.80$ to $-1.80, p<0.05$ ), $24 \mathrm{~h}$ ASBP (MD $-4.31 \mathrm{~mm} \mathrm{Hg}, 95 \% \mathrm{CI}-6.56$ to $-2.07, p<0.05$ ), and $24 \mathrm{~h}$ ADBP (MD $-1.69 \mathrm{~mm} \mathrm{Hg}, 95 \% \mathrm{CI}-2.59$ to $-0.79, p<0.05)$ when compared with ARBs. 
SBP

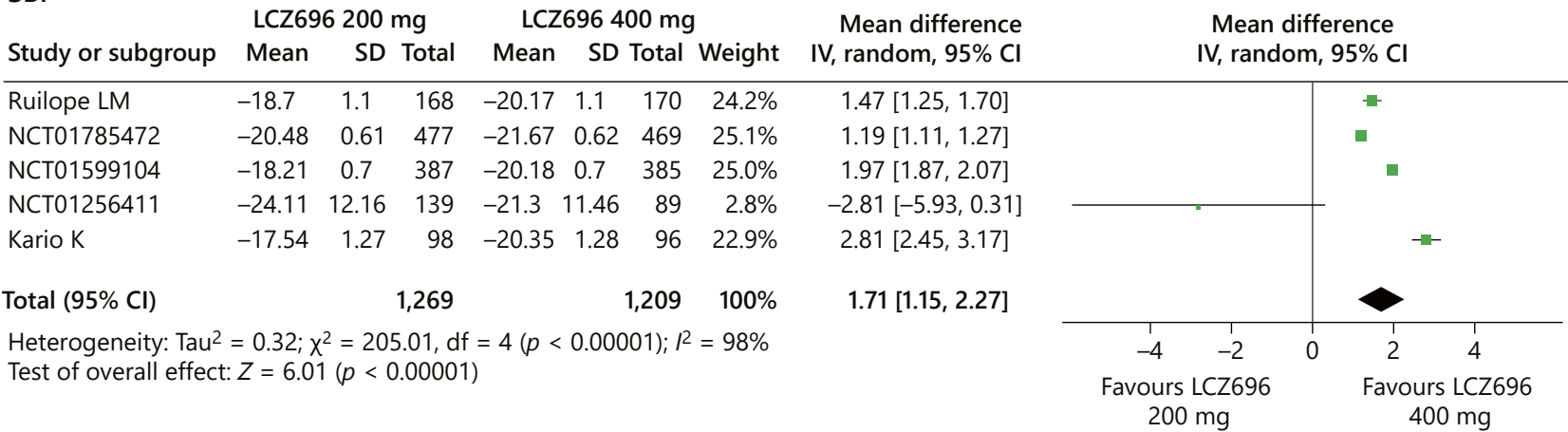

DBP

LCZ696 $200 \mathrm{mg} \quad$ LCZ696 $400 \mathrm{mg} \quad$ Mean difference

Study or subgroup Mean SD Total Mean SD Total Weight IV, random, $95 \% \mathrm{CI}$

\begin{tabular}{llllllll}
\hline Ruilope LM & -12.92 & 0.7 & 168 & -13.63 & 0.7 & 170 & $24.4 \%$
\end{tabular}

$\begin{array}{lllllllll}\text { NCT01785472 } & -8.1 & 0.37 & 477 & -8.8 & 0.38 & 469 & 26.1 \%\end{array}$

$\begin{array}{llllllll}\text { NCT01599104 } & -7.76 & 0.4 & 387 & -8.79 & 0.41 & 385 & 26.3 \%\end{array}$

$\begin{array}{llllllll}\text { NCT01256411 } & -16.6 & 7.63 & 139 & -14.2 & 7.05 & 89 & 1.6 \%\end{array}$

Kario K

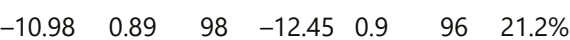

Total $(95 \% \mathrm{Cl})$

1,269

$1,209 \quad 100 \%$

$0.7[0.56,0.86]$

$0.7[0.65,0.75]$

$1.03[0.97,1.09]$

$-2.40[-4.34,-0.46]$

$1.47[1.22,1.72]$

Heterogeneity: $\mathrm{Tau}^{2}=0.06 ; \chi^{2}=113.10, \mathrm{df}=4(p<0.00001) ; R^{2}=96 \%$

Test of overall effect: $Z=6.93(p<0.00001)$

$0.90[0.65,1.16]$

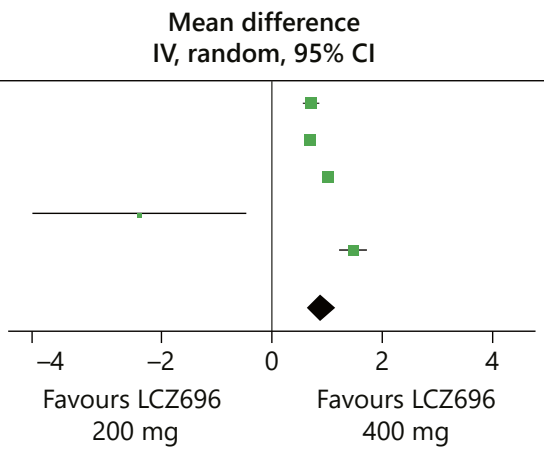

\section{$24 \mathrm{~h}$ ASBP}

Study or subgroup

LCZ696 $200 \mathrm{mg}$

LCZ696 $400 \mathrm{mg}$

\begin{tabular}{llllllll}
\hline Ruilope LM & -11.5 & 0.93 & 61 & -14.56 & 1.03 & 53 & $23.8 \%$
\end{tabular}

$\begin{array}{llllllll}\text { NCT01785472 } & -12.07 & 0.49 & 188 & -12.76 & 0.49 & 184 & 25.5 \%\end{array}$

$\begin{array}{llllllll}\text { NCT01599104 } & -13.44 & 0.45 & 216 & -14.99 & 0.45 & 216 & 25.6 \%\end{array}$

Kario K

$\begin{array}{lllllll}-15.18 & 0.64 & 84 & -15.98 & 0.65 & 82 & 25.1 \%\end{array}$

Total $(95 \% \mathrm{Cl})$

549

$535100 \%$

Heterogeneity: $\mathrm{Tau}^{2}=0.45 ; \chi^{2}=283.18, \mathrm{df}=3(p<0.00001) ; R^{2}=99 \%$

Test of overall effect: $Z=4.43(p<0.00001)$

Mean difference

IV, random, $95 \% \mathrm{Cl}$

$3.06[2.70,3.42]$

$0.69[0.59,0.79]$

$1.55[1.47,1.63]$

$0.80[0.60,1.00]$

$1.50[0.84,2.17]$

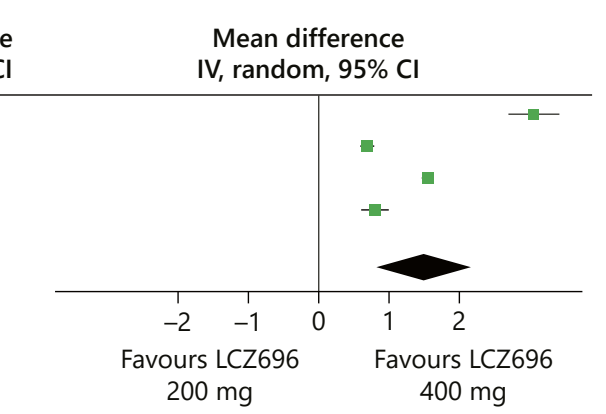

\section{$24 \mathrm{~h}$ ADBP}

Study or subgroup

Ruilope LM

NCT01785472

NCT01599104

Kario K

Total $(95 \% \mathrm{Cl})$

Heterogeneity: $\mathrm{Tau}^{2}=0.09 ; \chi^{2}=120.38, \mathrm{df}=3(p<0.00001) ; R^{2}=98 \%$

Test of overall effect: $Z=5.07(p<0.00001)$ Mean SD Total Weight

$\begin{array}{llll}-8.32 & 0.71 & 53 & 22.4 \%\end{array}$

$\begin{array}{llll}-6.82 & 0.32 & 184 & 26.3 \%\end{array}$

$\begin{array}{llll}-8.44 & 0.3 & 216 & 26.4 \%\end{array}$

$\begin{array}{llll}-9.69 & 0.5 \quad 82 \quad 24.9 \%\end{array}$

$\begin{array}{lll}-7.65 & 0.3 & 216\end{array}$

$\begin{array}{lll}-9.33 & 0.49 \quad 84\end{array}$

549

$535100 \%$
LCZ696 $200 \mathrm{mg} \quad$ LCZ696 $400 \mathrm{mg} \quad$ Mean difference

IV, random, $95 \% \mathrm{Cl}$

$1.54[1.29,1.79]$

$0.46[0.40,0.52]$

$0.79[0.73,0.85]$

$0.36[0.21,0.51]$

$0.76[0.47,1.06]$

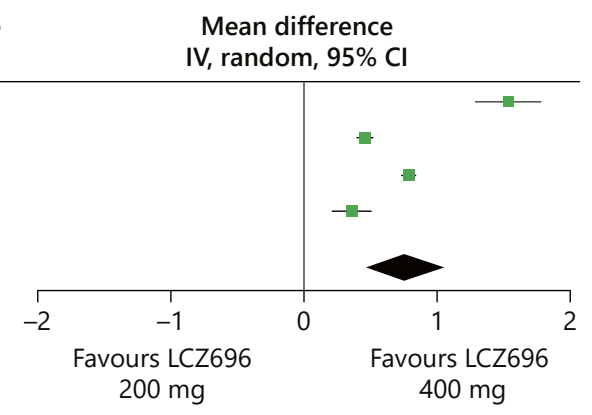

Fig. 6. Forest plots of SBP, DBP, $24 \mathrm{~h}$ ASBP, and $24 \mathrm{~h}$ ADBP in LCZ696 200 versus $400 \mathrm{mg}$. LCZ696 $400 \mathrm{mg}$ showed a significant reduction in SBP (MD $1.71 \mathrm{~mm} \mathrm{Hg}, 95 \%$ CI 1.15 to 2.27, $p<0.05$ ), DBP (MD $0.90 \mathrm{~mm} \mathrm{Hg}$, $95 \%$ CI 0.65 to $1.16, p<0.05$ ), $24 \mathrm{~h} \mathrm{ASBP}$ (MD $1.50 \mathrm{~mm} \mathrm{Hg}, 95 \%$ CI 0.84 to $2.17, p<0.05$ ), and $24 \mathrm{~h} \mathrm{ADBP}$ (MD $0.76 \mathrm{~mm} \mathrm{Hg}$, 95\% CI 0.47 to $1.06, p<0.05)$ when compared with LCZ696 $200 \mathrm{mg}$. 
tihypertensive drug. In addition to the cardiovascular benefits of RAS blockade, LCZ696 is capable of neprilysin inhibition, which enhances the reduction of blood pressure. Ruilope et al. [6] were the first to demonstrate the antihypertensive efficacy of LCZ696. Ye et al. [21] showed that LCZ696 is more effective in reducing blood pressure than placebo. Ito et al. [22] suggested that LCZ696 administration may be a promising therapeutic approach for Japanese patients with hypertension and renal dysfunction. In our meta-analysis, we found that LCZ696 (100, 200 , and $400 \mathrm{mg}$ ) produced significantly greater reductions in SBP, DBP, $24 \mathrm{~h}$ ASBP, and $24 \mathrm{~h} \mathrm{ADBP}$ than ARBs alone in hypertensive patients. These results are consistent with the meta-analyses of Ye et al. [21] and Zhao et al. [23]. Compared with ARBs alone, LCZ696 causes a greater reduction in blood pressure, mainly attributed to RAS inhibition occurring simultaneously with neprilysin inhibition.

Ours is the first meta-analysis to evaluate the efficacy of different LCZ696 dosages on blood pressure. We demonstrated the efficacy of LCZ696 $100 \mathrm{mg}$ versus $200 \mathrm{mg}$ and LCZ696 $200 \mathrm{mg}$ versus $400 \mathrm{mg}$. We found no significant differences in the reduction in SBP and DBP between 100 and $200 \mathrm{mg}$ of LCZ696. However, LCZ696 200 mg caused a significantly greater $24 \mathrm{~h}$ ASBP reduction than LCZ696 100 mg. 24 h ADBP did not differ significantly between 100 and $200 \mathrm{mg} \operatorname{LCZ696}(p=0.05)$. However, as shown in Figure 5, there was a tendency towards a reduction in $24 \mathrm{~h} \mathrm{ADBP}$ associated with $200 \mathrm{mg} \mathrm{LCZ696.}$ This result should be interpreted with caution. Largescale RCTs need to confirm the antihypertensive effects of 100 and $200 \mathrm{mg}$ LCZ696.

Compared with SBP and DBP, $24 \mathrm{~h}$ ASBP and $24 \mathrm{~h}$ ADBP are better able to reflect the antihypertensive effect of drugs. For patients with hypertension, we should pay more attention to 24 -h ambulatory blood pressure, as this is a better predictor of cardiovascular mortality and stroke than office blood pressure $[24,25]$. Mean plasma concentration time profiles for valsartan were found to be similar after the administration of LCZ696 $400 \mathrm{mg}$ or valsartan $320 \mathrm{mg}$ [26]. Mean terminal half-life (median $18 \mathrm{~h}$ ) was similar between LCZ696 $400 \mathrm{mg}$ and valsartan $320 \mathrm{mg}$ treatments. We found that $400 \mathrm{mg}$ LCZ696 caused significant reductions in SBP, DBP, $24 \mathrm{~h}$ ASBP, and $24 \mathrm{~h}$ ADBP compared with $200 \mathrm{mg}$ LCZ696. The guidelines recommended ARBs as the first-line therapy. LCZ696 is also effective in lowering blood pressure.

Several limitations of this meta-analysis should be noted. First, 5 of the included studies (NCT01353508, NCT01256411, NCT01599104, NCT01785472, and
NCT01876368) were not published in scientific articles and were derived from the Novartis Pharma database. This could affect the observed results. Second, most of the trials included had a short follow-up period. The reductions in blood pressure only reflected the short-term effects of LCZ696. Third, the types and dosages of ARBs were different; the olmesartan dosage was 20 or $40 \mathrm{mg}$ and the valsartan dosage was $80-320 \mathrm{mg}$. Fourth, the sample sizes in the LCZ696 $100 \mathrm{mg}$ and $200 \mathrm{mg}$ groups were very small. Additional large-scale studies are needed to confirm the effects of 100 and $200 \mathrm{mg}$ of LCZ696 on SBP, DBP, $24 \mathrm{~h}$ ASBP, and $24 \mathrm{~h} \mathrm{ADBP.}$

To summarize, LCZ696 was found to be safe and well tolerated [27]. This study provides new evidence for the reduction in blood pressure achieved with LCZ696 and adds new indications for LCZ696 in the instructions. The antihypertensive effect of LCZ696 is dose-related. LCZ696 can provide a reduction in blood pressure in addition to beneficial cardiovascular effects. For patients with heart failure and hypertension, treatment with LCZ696 should be given preference. Our results confirm the antihypertensive effects of LCZ696, a novel, dual-acting angiotensin receptor-neprilysin inhibitor.

\section{Highlights}

LCZ696 was found to be more effective in reducing blood pressure than ARBs. The antihypertensive effect of LCZ696 is dose-related. For heart failure complicated with hypertension, treatment with LCZ696 should be given preference.

\section{Author Contributions}

All authors had access to the data and participated in writing this manuscript.

References

1 Martin FL, Stevens TL, Cataliotti A, Schirger JA, Borgeson DD, Redfield MM, et al. Natriuretic and antialdosterone actions of chronic oral NEP inhibition during progressive congestive heart failure. Kidney Int. 2005 May; 67(5):1723-30.

2 Böhm M, Young R, Jhund PS, Solomon SD, Gong J, Lefkowitz MP, et al. Systolic blood pressure, cardiovascular outcomes and efficacy and safety of sacubitril/valsartan (LCZ696) in patients with chronic heart failure and reduced ejection fraction: results from PARADIGM-HF. Eur Heart J. 2017 Apr;38(15):1132-43. 
3 Yancy CW, Jessup M, Bozkurt B, Butler J, Casey DE Jr, Colvin MM, et al. 2016 ACC/ AHA/HFSA Focused Update on New Pharmacological Therapy for Heart Failure: An Update of the 2013 ACCF/AHA Guideline for the Management of Heart Failure: A Report of the American College of Cardiology/ American Heart Association Task Force on Clinical Practice Guidelines and the Heart Failure Society of America. J Am Coll Cardiol. 2016 Sep;68(13):1476-88.

4 Ponikowski P, Voors AA, Anker SD, Bueno H, Cleland JG, Coats AJ, et al.; Authors/Task Force Members; Document Reviewers. 2016 ESC Guidelines for the diagnosis and treatment of acute and chronic heart failure: the Task Force for the diagnosis and treatment of acute and chronic heart failure of the European Society of Cardiology (ESC). Developed with the special contribution of the Heart Failure Association (HFA) of the ESC. Eur J Heart Fail. 2016 Aug;18(8):891-975.

5 Moher D, Liberati A, Tetzlaff J, Altman DG; PRISMA Group. Preferred reporting items for systematic reviews and meta-analyses: the PRISMA statement. PLoS Med. 2009 Jul;6(7): e1000097.

6 Ruilope LM, Dukat A, Böhm M, Lacourcière Y, Gong J, Lefkowitz MP. Blood-pressure reduction with LCZ696, a novel dual-acting inhibitor of the angiotensin II receptor and neprilysin: a randomised, double-blind, placebo-controlled, active comparator study. Lancet. 2010 Apr;375(9722):1255-66.

7 Kario K, Sun N, Chiang FT, Supasyndh O, Baek SH, Inubushi-Molessa A, et al. Efficacy and safety of LCZ696, a first-in-class angiotensin receptor neprilysin inhibitor, in Asian patients with hypertension: a randomized, double-blind, placebo-controlled study. Hypertension. 2014 Apr;63(4):698-705.

8 Izzo JL Jr, Zappe DH, Jia Y, Hafeez K, Zhang J. Efficacy and Safety of Crystalline Valsartan/ Sacubitril (LCZ696) Compared with Placebo and Combinations of Free Valsartan and Sacubitril in Patients with Systolic Hypertension: the RATIO Study. J Cardiovasc Pharmacol. 2017 Jun;69(6):374-81.

9 Supasyndh O, Wang J, Hafeez K, Zhang Y, Zhang J, Rakugi H. Efficacy and Safety of Sacubitril/Valsartan (LCZ696) Compared with Olmesartan in Elderly Asian Patients $(\geq 65$ Years) With Systolic Hypertension. Am J Hypertens. 2017 Nov;30(12):1163-9.

10 Williams B, Cockcroft JR, Kario K, Zappe DH, Brunel PC, Wang Q, Guo W. Effects of sacubitril/valsartan versus olmesartan on central hemodynamics in the elderly with sys- tolic hypertension: the PARAMETER study. Hypertension. 2017;69:411-20.

11 Novartis Pharmaceuticals. [Internet] Efficacy and Safety of LCZ696 in Comparison to Olmesartan in Japanese Patients with Essential Hypertension. Bethesda, MD: National Institution of Health, Novartis Pharmaceuticals; 2012. Identifier NCT01599104. Available from: https://ClinicalTrials.gov/show/ NCT01599104.

12 Novartis Pharmaceuticals. [Internet] Efficacy and Safety of LCZ696 in Comparison to Olmesartan in Asian Patients with Essential Hypertension. Bethesda, MD: National Institution of Health, Novartis Pharmaceuticals; 2013. Identifier NCT01785472. Available from: https://ClinicalTrials.gov/show/ NCT01785472.

13 Novartis Pharmaceuticals. [Internet] Efficacy and Safety of LCZ696 Compared to Olmesartan in Essential Hypertensive Patients Not Responsive to Olmesartan. Bethesda, MD: National Institution of Health, Novartis Pharmaceuticals; 2013. Identifier NCT01876368. Available from: https://ClinicalTrials.gov/ show/ NCT01876368.

14 Schmieder RE, Wagner F, Mayr M, Delles C, Ott C, Keicher C, et al. The effect of sacubitril/ valsartan compared to olmesartan on cardiovascular remodelling in subjects with essential hypertension: the results of a randomized, double-blind, active-controlled study. Eur Heart J. 2017 Nov;38(44):3308-17.

15 Novartis Pharmaceuticals. [Internet] Sodium Excretion of LCZ696 in Patients with Hypertension; Heart Failure and Healthy Volunteers. Bethesda, MD: National Institution of Health, Novartis Pharmaceuticals; 2011. Identifier NCT01353508. Available from: https://ClinicalTrials.gov/show/ NCT01353508.

16 Wang TD, Tan RS, Lee HY, Ihm SH, Rhee MY, Tomlinson B, et al. Effects of Sacubitril/ Valsartan (LCZ696) on Natriuresis, Diuresis, Blood Pressures, and NT-proBNP in SaltSensitive Hypertension. Hypertension. 2017 Jan;69(1):32-41.

17 Novartis Pharmaceuticals. [Internet] A LongTerm (12 Months) Safety, Tolerability and Efficacy Study of LCZ696 in Patients with Essential Hypertension. Bethesda, MD: National Institution of Health, Novartis Pharmaceuticals; 2010. Identifier NCT01256411. Available from: https://ClinicalTrials.gov/ show/ NCT01256411.

18 McMurray JJ, Packer M, Desai AS, Gong J, Lefkowitz MP, Rizkala AR, et al.; PARADIGM-HF Investigators and Commit- tees. Angiotensin-neprilysin inhibition versus enalapril in heart failure. N Engl J Med. 2014 Sep;371(11):993-1004.

19 Solomon SD, Zile M, Pieske B, Voors A, Shah A, Kraigher-Krainer E, et al.; Prospective comparison of ARNI with ARB on Management Of heart failUre with preserved ejectioN fracTion (PARAMOUNT) Investigators. The angiotensin receptor neprilysin inhibitor LCZ696 in heart failure with preserved ejection fraction: a phase 2 double-blind randomised controlled trial. Lancet. 2012 Oct; 380(9851):1387-95.

20 Volpe M, Carnovali M, Mastromarino V. The natriuretic peptides system in the pathophysiology of heart failure: from molecular basis to treatment. Clin Sci (Lond). 2016 Jan;130(2): 57-77.

21 Ye L, Wang J, Chen Q, Yang X. LCZ696, a promising novel agent in treating hypertension (a meta-analysis of randomized controlled trials). Oncotarget. 2017 Nov;8(64): 107991-8005.

22 Ito S, Satoh M, Tamaki Y, Gotou H, Charney A, Okino N, et al. Safety and efficacy of LCZ696, a first-in-class angiotensin receptor neprilysin inhibitor, in Japanese patients with hypertension and renal dysfunction. Hypertens Res. 2015 Apr;38(4):269-75.

23 Zhao Y, Yu H, Zhao X, Ma R, Li N, Yu J. The Effects of LCZ696 in Patients with Hypertension Compared with Angiotensin Receptor Blockers: A Meta-Analysis of Randomized Controlled Trials. J Cardiovasc Pharmacol Ther. 2017 Sep;22(5):447-57.

24 Dolan E, Stanton A, Thijs L, Hinedi K, Atkins $\mathrm{N}, \mathrm{McClory}$ S, et al. Superiority of ambulatory over clinic blood pressure measurement in predicting mortality: the Dublin outcome study. Hypertension. 2005 Jul;46(1):156-61.

25 Clement DL, De Buyzere ML, De Bacquer DA, de Leeuw PW, Duprez DA, Fagard RH, et al.; Office versus Ambulatory Pressure Study Investigators. Prognostic value of ambulatory blood-pressure recordings in patients with treated hypertension. $\mathrm{N}$ Engl J Med. 2003 Jun;348(24):2407-15.

$26 \mathrm{Gu}$ J, Noe A, Chandra P, Al-Fayoumi S, Ligueros-Saylan M, Sarangapani R, et al. Pharmacokinetics and pharmacodynamics of LCZ696, a novel dual-acting angiotensin receptor-neprilysin inhibitor (ARNi). J Clin Pharmacol. 2010 Apr;50(4):401-14.

27 Geng Q, Li S, Wang Z, Ren Y. Efficacy and safety of combined neprilysin and RAS inhibition in heart failure: A meta-analysis of randomized controlled trials. Int J Cardiol. 2019 Oct;293:159-64. 